\title{
The chromospherically active, triple, ellipsoidal, and eclipsing binary HD 6286 = BE Piscium: a laboratory for binary evolution
}

\author{
K. G. Strassmeier ${ }^{1, \star, \star \star}$, J. Bartus ${ }^{1}$, F. C. Fekel ${ }^{2, \star}$, and G. W. Henry ${ }^{2}$
}

\author{
1 Astrophysical Institute Potsdam (AIP), An der Sternwarte 16, 14482 Potsdam, Germany \\ e-mail: [kstrassmeier; jbartus] @aip.de \\ 2 Center of Excellence in Information Systems, Tennessee State University, 3500 John A. Merritt Boulevard, Box 9501, Nashville, \\ Tennessee 37209, USA \\ e-mail: fekel@evans.tsuniv.edu;henry@schwab.tsuniv.edu
}

Received 6 December 2007 / Accepted 25 April 2008

\begin{abstract}
Aims. We present a detailed analysis of the star HD 6286 = BE Psc from 16 years of spectroscopic observations and 18 seasons of photometric ones. The star is an evolved, chromospherically active, eclipsing binary, consisting of a K1 giant plus an F6 dwarf/subgiant in a circular orbit with a period of 35.671 days. A faint, close visual companion of spectral type $\approx \mathrm{G} 0$ makes the system triple. The orbital inclination of the eclipsing pair is 81.8 .

Methods. We have obtained simultaneous solutions with our extensive set of radial velocities and $B V I$ light curves that include the star spot variability of the K giant, the ellipticity of the K giant, and the eclipses of the spectroscopic binary system.

Results. Our spot solutions suggest persistent polar spots, one in each hemisphere, that are cooler than the surrounding photosphere by $810 \pm 150 \mathrm{~K}$ over the timespan of our observations. The K giant and the F6 dwarf/subgiant have masses of $1.56 M_{\odot}$ and $1.31 M_{\odot}$ and mean radii of 12.0 and $1.9 R_{\odot}$, respectively. The masses have uncertainties of just $\approx 1.5 \%$. No irradiation effect was detected. We compared our results to theoretical evolutionary tracks that suggest an age for the system of $\approx 2.7$ Gyr. The modest logarithmic lithium abundance of the primary of 1.30 (upper limit) indicates that the star may have already experienced its first dredge up. The rotation period of the primary is $35.49 \pm 0.01$ days and appears to be synchronized with the orbital period of the eclipsing pair to within $0.5 \%$. Our data are inconclusive as to whether the secondary is synchronized.

Conclusions. Circularization of the orbit has taken place, and we conclude that the rapid increase in the size of the $\mathrm{K}$ giant, as it evolved across the Hertzsprung gap and up the base of the giant branch, likely caused the orbit to become circular.
\end{abstract}

Key words. stars: activity - stars: binaries: close - stars: binaries: spectroscopic - stars: binaries: visual - stars: starspots stars: binaries: eclipsing

\section{Introduction}

The late-type star HD $6286=$ BE Piscium $\left(\alpha=01^{\mathrm{h}} 04^{\mathrm{m}} 07^{\mathrm{s}}\right.$, $\delta=26^{\circ} 35^{\prime} 14^{\prime \prime}$ [2000]) was included in a spectroscopic survey carried out at the David Dunlap Observatory. From seven observations Heard (1956) found it to have a variable velocity, and the star was classified as G2 V. In 1969, Couteau (1970) discovered that this spectroscopic binary is part of a close visual binary, making the system triple. He measured a separation of 0 ' 6 , and estimated visual magnitudes of $8 . \cdot 3$ and $10^{\mathrm{m}} \cdot 0$ for the components. More recently, Fabricius \& Makarov (2000) used observations obtained with Hipparcos to provide more precise magnitudes. Reporting results from a northern-sky objectiveprism survey, Bidelman (1983) identified HD 6286 as a latetype star with moderate $\mathrm{Ca}$ II $\mathrm{H}$ and $\mathrm{K}$ emission, which prompted Strassmeier et al. (1993) to include the star in the second edition of the chromospherically active binaries catalog.

* Visiting Astronomer, Kitt Peak National Observatory, National Optical Astronomy Observatories, operated by the Association of Universities for Research in Astronomy, Inc. under cooperative agreement with the National Science Foundation.

$\star \star$ Visiting Astronomer, Canada-France-Hawaii Telescope, operated by the National Research Council of Canada, the Centre National de la Recherche Scientifique de France, and the University of Hawaii.
Because of its identification as a chromospherically active star, HD 6286 was included in various photometric and spectroscopic surveys. Hooten \& Hall (1990) collected three seasons of photometric observations and determined a period of 35.3 days. During those seasons they found that the amplitude of the light variation increased monotonically. As a result of their work, Kazarovets et al. (1993) assigned HD 6286 the variable star name BE Psc and classified it as a variable of the RS CVn type. From satellite observations the Hipparcos team (Perryman et al.; ESA 1997) obtained a photometric period of 35.53 days, in agreement with the value of Hooten \& Hall (1990). Pasquini \& Lindgren (1994) analyzed the Ca II K and $\mathrm{H} \alpha$ lines of 27 metalpoor binaries, including HD 6286. From their unpublished radial velocities they determined that HD 6286 has a nearly circular orbit with a period of 35.7 days and concluded that the primary is evolved. In their sample HD 6286 was the only star with a period greater than 10 days that had significant chromospheric emission. Crews et al. (1996) discovered HD 6286 to be an eclipsing binary and from our preliminary spectroscopic orbital elements gave an ephemeris for eclipses. As expected for a chromospherically active binary, the primary is rapidly rotating with $v \sin i=17.5 \mathrm{~km} \mathrm{~s}^{-1}$ (Fekel 1997). Although HD 6286 has not been included in radio surveys it has been detected at X-ray wavelengths (Dempsey et al. 1997). 


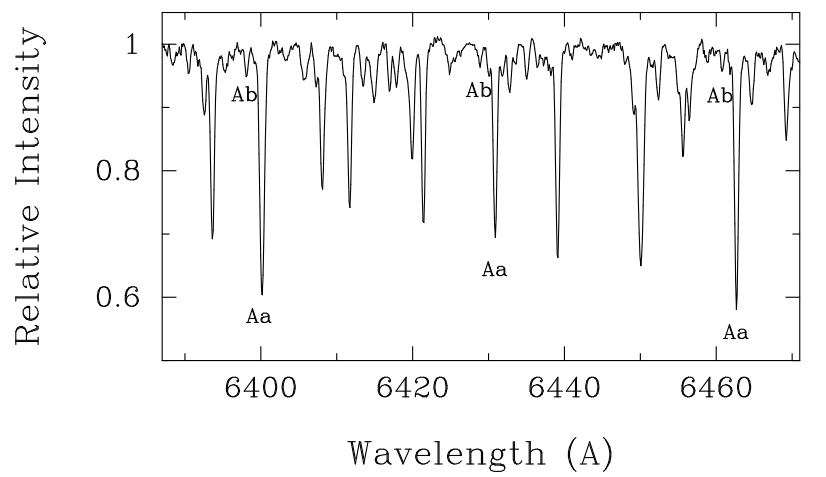

Fig. 1. Spectrum of HD 6286 in the $6430 \AA$ region. Three line pairs of component $\mathrm{Aa}$, the $\mathrm{K}$ giant primary, and component $\mathrm{Ab}$, the F-star secondary, are identified.

For 16 years we have acquired red-wavelength spectra of HD 6286; we have also acquired photometric observations covering 18 observing seasons. Our emphasis in this paper is on the basic properties of the system and its components and so, although we must account for the spot activity in our eclipse light curve, we do not analyze the long-term photometric behavior of the chromospherically active giant in all its possible detail. We compare our results with evolutionary tracks and discuss them in light of circularization and synchronization time scales.

\section{Observations and data reductions}

\subsection{Spectroscopy}

From 1989 October to 2005 June we obtained 61 high-resolution spectrograms, 14 of which were collected during a three-week observing run from 1997 December through 1998 January. The observations were made at the Kitt Peak National Observatory (KPNO) with the $0.9 \mathrm{~m}$ coudé feed telescope, coudé spectrograph, and a TI CCD detector. All of the spectrograms are centered in the red at $6430 \AA$, cover a wavelength range of about $80 \AA$, and have a resolving power $R$ of just over 30000 , corresponding to a resolution of $0.21 \AA$.

The radial velocities of the KPNO observations (Table 1) were determined with the IRAF cross-correlation program FXCOR (Fitzpatrick 1993). The cross-correlation reference stars were $\alpha$ Ari, $\beta$ Gem, HR 8551, 10 Tau, and $\iota$ Psc. All are IAU radial-velocity standards (Pearce 1957), and their velocities were adopted from the work of Scarfe et al. (1990). Some of the cross-correlation profiles of the primary showed asymmetries that result from a non-uniform distribution of star spots on that component. Lines of the secondary in the $6430 \AA$ region are very weak and often blended (Fig. 1). In spectra where the velocity separation between the primary and secondary is great enough, only one or two secondary lines proved useful for velocity measurement. At phases when the secondary is blue shifted, the least blended line is the combined Fe I and $\mathrm{Ca}$ I line at $6462.69 \AA$, while for red shifted phases the best line is the Ca I line at $6439.083 \AA$.

Five high-resolution spectra were obtained with the f/8 Gecko spectrograph at the $3.6 \mathrm{~m}$ Canada-France-Hawaii (CFH) telescope on Mauna Kea, Hawaii in 1997 October. Three observations were centered at $6710 \AA$, one at $6250 \AA$, and one at $6562 \AA$. The spectrograms covered approximately $60 \AA$ and have a resolving power of 120000 , corresponding to a resolution of $0.05 \AA$. All spectrograms were reduced with the standard
Table 1. Radial velocities, $v$, and residuals, $\left(\mathrm{O}-\mathrm{C}\right.$ ), in $\mathrm{km} \mathrm{s}^{-1}$ (HJD is $2400000+)$. The suffix $A a$ is for the primary and $A b$ is for the secondary.

\begin{tabular}{|c|c|c|c|c|c|}
\hline Hel. JD & Phase & $v_{\text {Aa }}$ & $(\mathrm{O}-\mathrm{C})_{\mathrm{Aa}}$ & $v_{\mathrm{Ab}}$ & $(\mathrm{O}-\mathrm{C})_{\mathrm{Ab}}$ \\
\hline 47809.832 & 0.514 & -69.3 & -1.7 & $\ldots$ & $\ldots$ \\
\hline 47810.926 & 0.545 & -68.1 & -2.0 & $\ldots$ & $\ldots$ \\
\hline 47811.927 & 0.573 & -65.0 & -1.5 & $\ldots$ & $\ldots$ \\
\hline 47812.950 & 0.601 & -60.7 & -1.1 & $\ldots$ & $\ldots$ \\
\hline 47813.958 & 0.630 & -55.8 & -1.2 & $\ldots$ & $\ldots$ \\
\hline 47814.936 & 0.657 & -49.8 & -0.8 & $\ldots$ & $\ldots$ \\
\hline 47815.932 & 0.685 & -43.1 & -0.6 & $\ldots$ & $\ldots$ \\
\hline 47916.679 & 0.509 & -68.5 & -0.8 & $\ldots$ & $\ldots$ \\
\hline 48572.724 & 0.901 & 9.2 & 1.0 & $\ldots$ & $\ldots$ \\
\hline 48605.745 & 0.826 & -6.7 & -0.2 & $\ldots$ & $\ldots$ \\
\hline 48913.869 & 0.464 & -66.9 & -0.2 & 20.6 & -2.2 \\
\hline 49245.876 & 0.771 & -20.3 & -0.1 & $\ldots$ & $\ldots$ \\
\hline 49248.825 & 0.854 & 0.4 & 0.7 & $\ldots$ & $\ldots$ \\
\hline 49249.842 & 0.883 & 6.4 & 1.2 & $\ldots$ & $\ldots$ \\
\hline 49250.806 & 0.910 & 10.6 & 1.1 & -67.9 & -0.0 \\
\hline 49301.788 & 0.339 & -47.7 & 0.4 & $\ldots$ & $\ldots$ \\
\hline 49618.876 & 0.228 & -21.7 & -1.6 & $\ldots$ & $\ldots$ \\
\hline 49619.977 & 0.259 & -28.1 & 0.1 & $\ldots$ & $\ldots$ \\
\hline 49621.934 & 0.314 & -42.0 & 0.2 & $\ldots$ & $\ldots$ \\
\hline 49674.875 & 0.798 & -13.7 & -0.3 & $\ldots$ & $\ldots$ \\
\hline 49677.830 & 0.881 & 6.1 & 1.2 & $\ldots$ & $\ldots$ \\
\hline 49968.949 & 0.042 & 14.7 & 0.0 & -72.9 & 1.1 \\
\hline 49969.968 & 0.070 & 11.6 & -0.5 & -70.2 & -0.7 \\
\hline 49970.857 & 0.095 & 8.2 & -0.6 & -68.3 & -1.3 \\
\hline 49971.866 & 0.124 & 3.3 & -0.8 & $\ldots$ & $\ldots$ \\
\hline 49972.965 & 0.154 & -3.5 & -1.4 & $\ldots$ & $\ldots$ \\
\hline 49973.738 & 0.176 & -8.2 & -1.2 & $\ldots$ & $\ldots$ \\
\hline 50265.984 & 0.369 & -52.5 & 1.8 & $\ldots$ & $\ldots$ \\
\hline 50266.969 & 0.396 & -57.9 & 1.3 & 15.0 & 1.1 \\
\hline 50361.917 & 0.058 & 11.3 & -2.0 & -73.1 & -0.7 \\
\hline 50809.761 & 0.613 & -57.3 & 0.4 & $\ldots$ & $\ldots$ \\
\hline 50810.760 & 0.641 & -51.4 & 1.0 & $\ldots$ & $\ldots$ \\
\hline 50811.682 & 0.667 & -45.8 & 1.0 & $\ldots$ & $\ldots$ \\
\hline 50812.592 & 0.692 & -39.9 & 0.8 & $\ldots$ & $\ldots$ \\
\hline 50813.653 & 0.722 & -33.0 & 0.2 & $\ldots$ & $\ldots$ \\
\hline 50814.695 & 0.751 & -24.5 & 1.0 & $\ldots$ & $\ldots$ \\
\hline 50815.714 & 0.780 & -18.6 & -0.6 & $\ldots$ & $\ldots$ \\
\hline 50819.744 & 0.893 & 7.5 & 0.6 & $\ldots$ & $\ldots$ \\
\hline 50820.703 & 0.920 & 11.7 & 0.8 & $\ldots$ & $\ldots$ \\
\hline 50821.701 & 0.948 & 14.5 & 0.6 & $\ldots$ & $\ldots$ \\
\hline 50825.738 & 0.061 & 11.9 & -1.2 & $\ldots$ & $\ldots$ \\
\hline 50826.732 & 0.089 & 9.3 & -0.5 & $\ldots$ & $\ldots$ \\
\hline 50827.730 & 0.117 & 5.1 & -0.3 & $\ldots$ & $\ldots$ \\
\hline 50828.728 & 0.145 & -0.2 & -0.2 & $\ldots$ & $\ldots$ \\
\hline 50829.656 & 0.171 & -5.0 & 0.8 & $\ldots$ & $\ldots$ \\
\hline 50832.641 & 0.254 & -24.2 & 2.8 & $\ldots$ & $\ldots$ \\
\hline 51473.819 & 0.229 & -20.0 & 0.3 & $\ldots$ & $\ldots$ \\
\hline 51474.775 & 0.256 & -26.0 & 1.3 & $\ldots$ & $\ldots$ \\
\hline 51475.846 & 0.286 & -33.1 & 2.0 & $\ldots$ & $\ldots$ \\
\hline 51476.832 & 0.313 & -40.6 & 1.5 & $\ldots$ & $\ldots$ \\
\hline 51802.863 & 0.453 & -65.1 & 0.9 & 21.7 & 0.2 \\
\hline 52179.862 & 0.022 & 15.4 & -0.3 & -74.6 & 0.6 \\
\hline 52180.850 & 0.049 & 14.1 & 0.0 & -73.9 & -0.6 \\
\hline 52181.893 & 0.079 & 10.8 & -0.3 & -71.4 & -1.7 \\
\hline 52536.871 & 0.030 & 14.7 & -0.7 & -72.5 & 2.3 \\
\hline 52537.865 & 0.058 & 13.2 & -0.2 & -72.1 & 0.3 \\
\hline 52538.849 & 0.086 & 10.2 & 0.0 & -70.7 & -2.0 \\
\hline 52539.853 & 0.114 & 6.4 & 0.5 & -62.1 & 1.4 \\
\hline 52540.859 & 0.142 & 1.0 & 0.5 & & \\
\hline 53533.968 & 0.982 & 16.0 & 0.1 & -76.0 & -0.6 \\
\hline 53535.968 & 0.038 & 16.3 & 1.4 & -76.0 & -1.7 \\
\hline
\end{tabular}




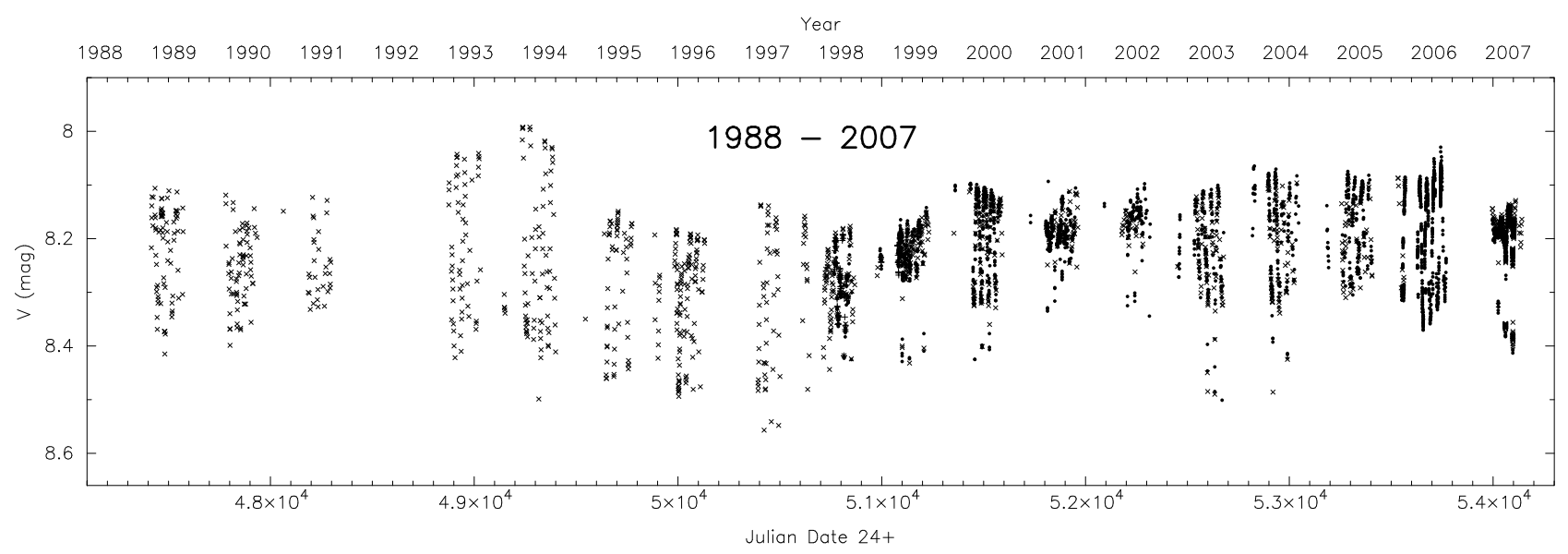

Fig. 2. Long-term $V$ light curve of HD 6286. The plot shows APT data covering 18 observing seasons between 1988/1989 and 2006/2007 but without data from 1991/1992. All observations were acquired with three automatic telescopes (T3, T6, and T7) at Fairborn Observatory. Crosses are T3 data, dots are T7 data, and pluses are the T6 Strömgren $y$ data. The rotational modulation with a period of $\approx 35$ days is easily seen in the plot. The offset points in the lower envelope of the plot are eclipse points. Note that the system was brightest in 1993/1994 with a combined $V$ magnitude of 7.99 and faintest in 1996/97 during an eclipse of 8. 57.

IRAF setup for coudé spectra, following the procedure described in Strassmeier \& Rice (1998).

\subsection{Photometry}

Our photometric observations cover 18 observing seasons spanning the 19 years from 1988 to 2007. The observations were obtained with three different automatic photoelectric telescopes (APTs), all located at Fairborn Observatory near Washington Camp, Arizona. Nightly observations of HD 6286 began with the TSU T3 $0.4 \mathrm{~m}$ APT during the 1988/1989 observing season. Except for the 1991/1992 season, when the T3 photometer and control system were being upgraded, the T3 APT collected nightly observations every year through the 2006/2007 observing season. In 1997 the two University of Vienna/Astrophysical Institute Potsdam (AIP) $0.75 \mathrm{~m}$ APTs, T6 and T7, joined in the mission on a three data points per night basis. The T3, T6, and T7 APTs have provided a total of 1881 Johnson $B$ and $V$, 109 Strömgren $b$ and $y$, and 2474 Johnson $V$ and $I$ measurements, respectively. The data reduction and transformation procedures for T3 were described by Henry et al. (2005) and for T6 and T7 by Granzer et al. (2001). For details, we refer the reader to those papers and references therein.

Figure 2 shows the combined $V$-band data set for the $18 \mathrm{ob}-$ serving seasons. The 109 Strömgren $y$ observations from T6 are also plotted as $V$ points, as is common practice since the $V$ and $y$ bands have the same effective wavelength. Thus, we have a total $4464 \mathrm{~V}$ magnitudes, each of which is derived from the mean of generally three successive differential magnitudes between HD 6286 and a comparison star. The T6 and T7 APTs used HD 6274 (G0) and HD 6133 (F2) as comparison and check stars, respectively. Both telescopes reached approximately one million counts for integrations of 60 and $20 \mathrm{~s}$ for T6 and T7, respectively. The T3 APT observations were obtained with different comparison and check stars, HD 6009 (G8) and HD 5516 (G8), respectively. All differential magnitudes were converted to standard magnitudes with the help of an all-sky solution of the T7 data (see Granzer et al. 2001). Observations from T3 and T6 were shifted to match the T7 data with shifts derived from the phased 1997/1998 and 1998/1999 observing seasons, when observations from all three telescopes were available. The resulting comparison star magnitudes for T3 and T6 were $V=6.710$ and $V=8$. 988 , respectively.

\section{Spectrum appearance}

Initial spectral types of HD 6286 were determined with the spectrum-addition procedure of Strassmeier \& Fekel (1990). They identified several luminosity-sensitive and temperaturesensitive line ratios in the 6430-6465 $\AA$ region and used them, along with the general appearance of the spectrum, as spectraltype criteria. The spectra of late- $\mathrm{G}$ and early-K subgiant and giant reference stars from the list of Keenan \& McNeil (1989) and F stars from Fekel (1997) were used for comparison. Those spectra were obtained at KPNO with the same telescope, spectrograph and detector as our spectra of HD 6286. Comparison spectra were created with a computer program developed by Huenemoerder \& Barden (1984) and Barden (1985). Various combinations of reference star spectra were rotationally broadened, shifted in radial velocity, appropriately weighted, and added together to compare with the observed spectra of HD 6286.

Lines of the primary dominate the $6430 \AA$ region, so its spectral type was determined first. The best fit was with $\beta$ Gem, which has a K0 IIIb spectral type (Keenan \& McNeil 1989) and mean $[\mathrm{Fe} / \mathrm{H}]=-0.00$ (Taylor 1999). As noted above, lines of the secondary are quite weak, making accurate classification of it difficult. When combined with the spectrum of $\beta \mathrm{Gem}$, spectra of HR 5075 (F2 V, Abt \& Morrell 1995, and $[\mathrm{Fe} / \mathrm{H}]=-0.04$ Boesgaard \& Tripicco 1986) or Procyon (F5 IV-V, Johnson \& Morgan 1953, and $[\mathrm{Fe} / \mathrm{H}]=-0.02$ Taylor 2003) provided better fits than stars with F0 or late-F spectral classes. For F stars the lines in this wavelength region are not luminosity sensitive, so it is not possible to differentiate between dwarf and subgiant. Thus, our best spectral classification for HD 6286 is K0 III + F2-5. We find no clear evidence of lines from the visual secondary (the B component), which is about 1.9 mag fainter than the combined light of the spectroscopic binary components (Fabricius \& Makarov 2000). Also, we did not correct for the tertiary continuum dilution because we are interested in a first spectral-type estimate for the primary. The reference stars have near solar abundances, and thus, HD 6286 does as well, so the system is not 
very metal poor, as suggested by Pasquini \& Lindgren (1994), who listed $[\mathrm{Fe} / \mathrm{H}]=-1.4$.

We have remeasured $22 \mathrm{KPNO} R=30000$ spectra and obtained $v \sin i=18.1 \pm 1.0 \mathrm{~km} \mathrm{~s}^{-1}$, and five CFHT $R=$ 120000 spectra and obtained $18.9 \pm 0.3 \mathrm{~km} \mathrm{~s}^{-1}$, in fair agreement with the $17.5 \pm 1.0 \mathrm{~km} \mathrm{~s}^{-1}$ published earlier by Fekel (1997). A macroturbulence of $3 \mathrm{~km} \mathrm{~s}^{-1}$, as suggested by Fekel (1997), was adopted. Although we have subtracted the secondary and the tertiary continua from the single-epoch $R=120000$ CFHT spectra (shown later in Fig. 7), the primary lines remain fully blended with the weak secondary lines because all CFHT spectra were taken near a time of conjunction. Therefore, $v \sin i$ from these spectra is less reliable despite the higher spectral resolution and the smaller internal error.

\section{Spectroscopic orbit}

Our second step was to analyze the 61 KPNO radial velocities of the primary. Using the orbital period of Pasquini \& Lindgren (1994), we first computed preliminary orbital elements with BISP, a computer program that uses a slightly modified version of the Wilsing-Russell method (Wolfe et al. 1967). We refined those elements with a differential corrections program (Barker et al. 1967), called SB1. With weights of 0.01 we then included the seven radial velocities obtained at the David Dunlap Observatory (DDO) (Heard 1956) in the next orbital solution. The only orbital element with improved precision was the period, which had a value of $35.6715 \pm 0.0006$ days. However, this period from the combined data solution was not significantly different from the one obtained with the KPNO velocities alone, and so we adopted the KPNO solution. Because the eccentricity of that solution was small, $0.019 \pm 0.004$, we computed a circular orbit with SB1C (Barlow 1998, private communication), which also uses differential corrections to determine the elements. According to the precepts of Lucy \& Sweeney (1971), the eccentric orbit is to be preferred over the circular one. However, velocities obtained on several of our extended observing runs show systematic residuals, suggesting that the spot distribution affects the velocities of the primary. Thus, we have chosen to adopt a circular orbit, which is supported by the photometric observations. For our final solution, 17 secondary velocities, given weights of 0.5 each, were included with the primary velocities, and a double lined circular-orbit solution of the KPNO velocities (Table 2) was computed with SB2C, a modified version of SB1C. For a circular orbit the element $T$, a time of periastron passage, is undefined and so, as recommended by Batten et al. (1989), $T_{0}$, a time of maximum velocity for the primary, is given instead. Table 1 lists the fractional phases, referenced to that epoch, and the velocity residuals determined from the circular orbit fit. Figure 3 compares the velocities with the computed velocity curve. Zero phase is a time of maximum radial velocity of the primary.

\section{Combined photometric and spectroscopic solution}

\subsection{Third light}

HD 6286 is a close visual binary with a separation of 0.7 (Perryman et al. 1997) for which the Tycho catalog gives separate magnitudes for the A and B components (Fabricius \& Makarov 2000). Transformation of the Tycho $B$ and $V$ magnitudes results in $V=8 \mathrm{~m} .43$ and $B-V=1.01$ mag for component A, the eclipsing pair, and $V=10 \mathrm{~m} 35$ and $B-V=0.58$
Table 2. Spectroscopic orbital elements.

\begin{tabular}{ll}
\hline \hline Parameter & Value \\
\hline$P($ days $)$ & $35.67142 \pm 0.00080$ \\
$T_{0}(\mathrm{HJD})$ & $2449254.028 \pm 0.033$ \\
$\gamma\left(\mathrm{km} \mathrm{s}^{-1}\right)$ & $-25.83 \pm 0.14$ \\
$K_{\mathrm{Aa}}\left(\mathrm{km} \mathrm{s}^{-1}\right)$ & $41.94 \pm 0.19$ \\
$K_{\mathrm{Ab}}\left(\mathrm{km} \mathrm{s}^{-1}\right)$ & $49.87 \pm 0.41$ \\
$e$ & $0.0($ adopted $)$ \\
$a_{\mathrm{Aa}} \sin i\left(10^{6} \mathrm{~km}\right)$ & $20.575 \pm 0.095$ \\
$a_{\mathrm{Ab}} \sin i\left(10^{6} \mathrm{~km}\right)$ & $24.464 \pm 0.202$ \\
$m_{\mathrm{Aa}} \sin ^{3} i\left(M_{\odot}\right)$ & $1.558 \pm 0.028$ \\
$m_{\mathrm{Ab}} \sin ^{3} i\left(M_{\odot}\right)$ & $1.310 \pm 0.017$ \\
\hline
\end{tabular}

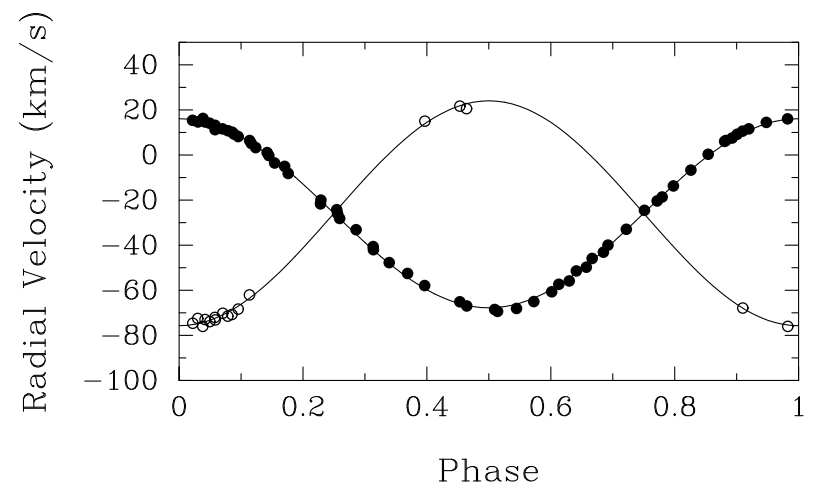

Fig. 3. A plot of the observed radial velocities compared with the computed velocity curve. Filled circles $=$ component Aa, open circles $=$ component Ab. Solid lines were computed from the elements in Table 2. Zero phase is a time of maximum velocity of the primary.

for component B. The small angular separation of the visual pair means that our photometric and spectroscopic observations always included both visual components in the $30^{\prime \prime}$ diaphragm and the $280 \mu \mathrm{m}$ slit width, respectively. Thus, the light curves and the spectra contain a contribution from the visual secondary. It causes dilution of the eclipses and of the spectral lines.

The visual companion was assumed to be constant in its light output, and so an intrinsic magnitude difference of 1.8 , based on the 1.9 given by Fabricius \& Makarov (2000), was adopted for third light. The $0^{\mathrm{m}} 1$ difference occurs because the Hipparcos magnitude of $\mathrm{Aa}+\mathrm{Ab}$ was observed at a different time and had to be corrected for the changing light level of the spot contribution.

\subsection{Rotational period of the primary}

The $V$-band data from the three APTs combine to produce a time series covering 18 observing seasons. With the eclipse points removed a total of 3918 data points was available for an out-ofeclipse period search. Because most seasons show a sinusoidal light curve (see Fig. 5), a simple Fourier analysis seemed appropriate. We applied the program package MUFRAN (Kolláth 1990), which is a collection of methods for analyzing multi periodic and unevenly sampled data. Standard discrete Fourier transforms of the light-curves were calculated, followed by a nonlinear least-square fit of the frequencies found. The combined $V$ and $y$ data were used, and a period of $35.49 \pm 0.01$ days resulted in the lowest residuals. This period differs by just $0.5 \%$ from the orbital period, and we interpret it as the average rotational period of the primary. For the spot analysis, we chose the (more stable) orbital period to phase all data in this paper. 


\subsection{Binary and stellar modelling}

\subsubsection{Quasi simultaneous solution}

Our first approach was to solve quasi-simultaneously for the orbital, stellar, and spot parameters. This seemed mathematically more consistent than a subsequent subtraction procedure (rectification), where one effect after another is removed from the observed data (e.g. Oláh et al. 1994). However, neither PHOEBE (Prsa \& Zwitter 2005) nor Nightfall (Wichmann 2006) was designed for complex multi-color spot modelling, and the goodness of eclipse fits was limited by the implicit spot-model assumptions and the numerical set-up of these codes that included spots. An additional problem is the non-uniqueness of the spot area and spot temperature as a function of wavelength (discussed in Sect. 5.4).

Our quasi-simultaneous approach, dubbed SIM, is only applied to the well-covered 1999/2000 observing season but enables us to obtain a full compliment of the system's orbital and stellar parameters. The season shows a comparatively stable light curve for the four stellar rotations covered (both by T3 in $B V$ and $\mathrm{T} 7$ in $V I$ ) and is thus well suited for such a representative task. Another season, 2006/2007, was optimized for extensive coverage of the two eclipses by T3 but, unfortunately, the spot activity varied significantly during this season.

We used the PHOEBE program to solve for the system and component parameters and also independently employed the Nightfall program. Both programs are based on a physical model of the binary that takes into account non-spherical shape as well as irradiance effects and surface spots. Neither of these codes performs a full error propagation, and thus, the programs do not output error bars for individual quantities. However, we estimate these, at least to first order, by comparing the consistency of the output from the two codes. Our input consisted of the radialvelocity data (Table 1) and the respective seasonal light curves, either $B V$ or $V I$ or combinations thereof, solved simultaneously. The visual companion, component $\mathrm{B}$, was assumed to be constant in its light output.

Table 3 lists the PHOEBE solution in column SIM. The differences between Nightfall and PHOEBE results provide an estimate of the internal uncertainties for each stellar parameter. The results from both codes agreed very well, even for the surface gravities where a difference of $<0.1$ dex was seen; $\log g=2.51$ and 2.49 for the primary and $\log g=4.01$ and 3.98 for the secondary (in cgs units) for Nightfall and PHOEBE, respectively. Both programs produce the same inclination of 81.8 , which is also used to determine the final radii, full masses and other $\sin i$-dependent parameters from the double-lined solution in Table 2. The surface potential of the primary indicates that the star fills $\approx 50 \%$ of its Roche lobe. This causes an ellipticity effect with a peak-to-peak magnitude of 0.015 in $V$. The secondary fills only $\approx 8 \%$ of its Roche lobe.

A word of caution is in order. The agreement of the quasisimultaneous (combined) solutions should not be over interpreted. The number of light-curve data points during eclipse is always small (see Fig. 4), but it is these data points that are crucial in determining the radii and light ratios. The duration of the eclipses translates into $R_{\mathrm{Aa}}+R_{\mathrm{Ab}}=9.0 \pm 0.2 R_{\odot}$ and drives the solution for the individual component's other absolute quantities such as $T_{\text {eff }}, \log g$, and mass, as listed in Table 3. Reasonable external uncertainties are not easily determined because of this undersampling of eclipse points. Moreover, the out-of-eclipse light variations due to spots are comparable or even larger than the primary eclipse depth. Although we have formally solved simultaneously for the spot distribution, the eclipse light curve, and
Table 3. Combined (B)VI-light curve and velocity curve results from Sect. 5.3.

\begin{tabular}{|c|c|c|}
\hline Parameter & SIM & REC \\
\hline Period (days) & 35.671 & 35.671 \\
\hline Eccentricity & 0.0 & 0.0 \\
\hline Mean Temperature of Aa $(\mathrm{K})$ & 4710 & 4500 \\
\hline Mean Temperature of $\mathrm{Ab}(\mathrm{K})$ & 6440 & 6300 \\
\hline$L_{\mathrm{Aa}} /\left(L_{\mathrm{Aa}+\mathrm{Ab}+\mathrm{B}}\right)$ in $B$ & $\ldots$ & 72.7 \\
\hline$L_{\mathrm{Aa}} /\left(L_{\mathrm{Aa}+\mathrm{Ab}+\mathrm{B}}\right)$ in $V$ & 79.0 & 79.2 \\
\hline$L_{\mathrm{Aa}} /\left(L_{\mathrm{Aa}+\mathrm{Ab}+\mathrm{B}}\right)$ in $I$ & 85.4 & 85.1 \\
\hline$L_{\mathrm{Ab}} /\left(L_{\mathrm{Aa}+\mathrm{Ab}+\mathrm{B}}\right)$ in $B$ & & 14.1 \\
\hline$L_{\mathrm{Ab}} /\left(L_{\mathrm{Aa}+\mathrm{Ab}+\mathrm{B}}\right)$ in $V$ & 11.0 & 10.8 \\
\hline$L_{\mathrm{Ab}} /\left(L_{\mathrm{Aa}+\mathrm{Ab}+\mathrm{B}}\right)$ in $I$ & 6.9 & 7.2 \\
\hline$L_{\mathrm{B}} /\left(L_{\mathrm{Aa}+\mathrm{Ab}+\mathrm{B}}\right)$ in $B$ & & 13.2 \\
\hline$L_{\mathrm{B}} /\left(L_{\mathrm{Aa}+\mathrm{Ab}+\mathrm{B}}\right)$ in $V$ & 10.0 & 10.0 \\
\hline$L_{\mathrm{B}} /\left(L_{\mathrm{Aa}+\mathrm{Ab}+\mathrm{B}}\right)$ in $I$ & 7.7 & 7.7 \\
\hline Inclination (deg) & 81.8 & 81.8 \\
\hline$R V$ semiamplitude, $\mathrm{Aa}\left(\mathrm{km} \mathrm{s}^{-1}\right)$ & 41.52 & 41.52 \\
\hline$R V$ semiamplitude, $\mathrm{Ab}\left(\mathrm{km} \mathrm{s}^{-1}\right)$ & 49.43 & 49.43 \\
\hline Mass Ratio, Ab/Aa & 0.840 & 0.840 \\
\hline Semimajor Axis $\left(10^{6} \mathrm{~km}\right)$ & 45.08 & 45.08 \\
\hline Semimajor Axis $\left(R_{\odot}\right)$ & 64.76 & 64.76 \\
\hline Surface Gravity, Aa (log,cgs) & 2.5 & 2.5 \\
\hline Surface Gravity, Ab (log,cgs) & 4.0 & 4.0 \\
\hline Mass, $\mathrm{Aa}\left(M_{\odot}\right)$ & 1.56 & 1.56 \\
\hline Mass, $\mathrm{Ab}\left(M_{\odot}\right)$ & 1.31 & 1.31 \\
\hline Polar Radius, $\mathrm{Aa}\left(R_{\odot}\right)$ & 11.75 & 11.98 \\
\hline Point Radius, $\mathrm{Aa}\left(R_{\odot}\right)$ & 11.82 & 12.17 \\
\hline Mean Radius, $\mathrm{Aa}\left(R_{\odot}\right)$ & 11.48 & 12.01 \\
\hline Mean Radius, $\mathrm{Ab}\left(R_{\odot}\right)$ & 1.94 & 1.88 \\
\hline Roche Filling Factor, Aa (\%) & 49 & 50 \\
\hline Roche Filling Factor, $\mathrm{Ab}(\%)$ & 8 & 8 \\
\hline Gravity Darkening, Aa & 0.21 & 0.10 \\
\hline Gravity Darkening, $\mathrm{Ab}$ & 0.25 & 0.08 \\
\hline Limb Darkening, Aa in $V$ & 0.76 & 0.80 \\
\hline Limb Darkening, Aa in I & 0.51 & 0.53 \\
\hline Limb Darkening, $\mathrm{Ab}$ in $V$ & 0.53 & 0.55 \\
\hline Limb Darkening, $\mathrm{Ab}$ in $I$ & 0.35 & 0.36 \\
\hline
\end{tabular}

Note: SIM is the quasi-simultaneous solution with just the 1999/2000 data set, REC is the iteratively rectified solution from all observing seasons combined. REC is our preferred solution. $L$ is the luminosity ratio and $R V$ the radial velocity. The period and the eccentricity were fixed at the values obtained from the orbit.

the radial velocities, some uncertainties will remain due to spot fits of only moderate quality. We conclude, that the rectifiedsolution approach in the next section provides a more consistent and likely better binary model.

\subsubsection{Rectified solution}

For our final analysis, we constructed rectified light curves and solved iteratively for the binary and stellar parameters and the spot parameters, respectively. All iterations were done on dereddened light curves with the third component removed (based on the values in Table 5). The PHOEBE SIM solution provided starting parameters for the binary model. This initial model was first removed from the 1999/2000 light curves based on Eq. (1),

$F_{\text {spot }}=F_{\text {obs }}-F_{\mathrm{Aa}+\mathrm{Ab}}^{0}-F_{\mathrm{Aa}+\mathrm{Ab}}^{\text {binary }}+F_{\mathrm{Aa}}^{0}$,

and the remaining light curve was solved simultaneously in $V$ and $I$ with the program SPOTMODEL (Ribarik et al. 2003). Here, the spot contribution is denoted $F_{\text {spot }}$, the observations are $F_{\text {obs }}$, the binary unspotted flux is $F_{\mathrm{Aa}+\mathrm{Ab}}^{0}$, the binary model is $F_{\mathrm{Aa}+\mathrm{Ab}}^{\text {binary }}$, and the unspotted flux from the spotted Aa component is $F_{\mathrm{Aa}}^{0}$. 

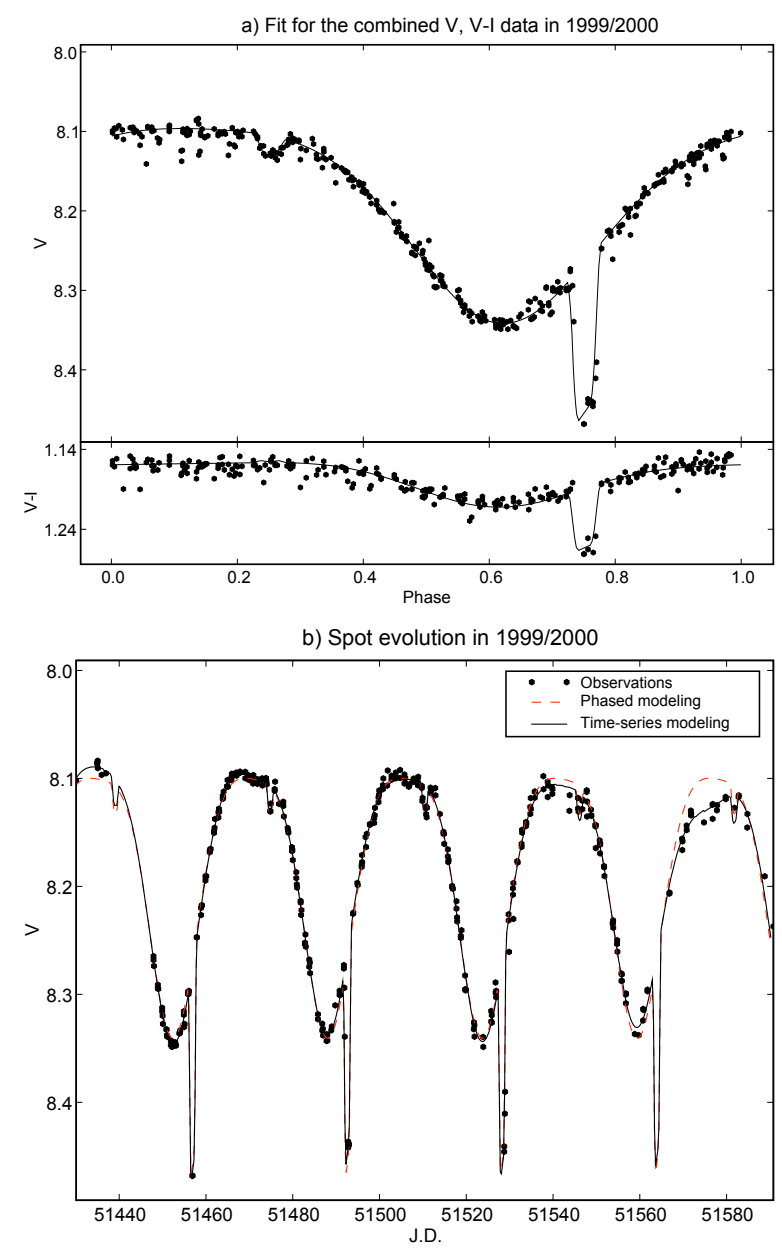

Fig. 4. a) The $\mathrm{T} 3+\mathrm{T} 7$ seasonal $1999 / 2000$ de-reddened $V$ band data (dots), chosen for the initial light curve solution, and the computed REC model fit (solid line). b) The same data as above but versus Julian date. The dashed line is simply the fit from panel a), which has been repeated for four cycles. The solid line is a fit to the time series with SpotModeL. It shows that at the end of the season the star spot distribution changed.

Two spots were adopted with the assumption that they have the same effective temperature. The geometrical parameters of the spots (position and size) and a single spot temperature were the free parameters. It was found that by slightly changing the unspotted $I$ magnitude of the Aa component, a significantly better $V I$ fit could be achieved. The best unspotted $I$ magnitude of $\mathrm{Aa}+\mathrm{Ab}$, adopted from the least squares of the residuals, was 6 . 854 with an overall uncertainty of $\approx 1 \%$. After this best fit was established, the spot solution was removed from the observed light curves and the binary model was improved with a simultaneous VI fit to the rectified data with Nightfall following again Eqs. (1) and (2),

$F_{\mathrm{Aa}}^{0}=F_{\mathrm{Aa}+\mathrm{Ab}}^{0} \frac{F_{\mathrm{Aa}} / F_{\mathrm{Ab}}}{1+F_{\mathrm{Aa}} / F_{\mathrm{Ab}}}$.

This process was then iterated until no more improvements were achieved. The unspotted flux of component $\mathrm{Aa}, F_{\mathrm{Aa}}^{0}$, is obtained from the flux ratio $\mathrm{Aa} / \mathrm{Ab}$ at the given wavelength, as iterated from the (combined) VI-light curves and radial-velocity solution. Then, the same procedure was repeated for all the other observing seasons that had $V$ and $I$ light curves (T7) and where a spot-temperature determination was possible (see the entries in Table 4). The seven spot-rectified light curves were then combined into a single "binary"-light curve and again fitted with Nightfall to get an even further improved binary solution. Finally, this second iteration series was repeated until no more improvements were possible in all of the observing seasons that had high-quality VI data. This solution is adopted as our final solution in column REC in Table 3. The main difference between the two approaches, SIM vs. REC, is that the rectified version converges at $\mathrm{a} \approx 4 \%$ larger and cooler Aa component because of a better fit to the spot contribution.

Figures $4 \mathrm{a}$ and $4 \mathrm{~b}$ show the $V I$ band data versus rotational phase and Julian date for the 1999/2000 season, along with the respective REC fits (the radial-velocity solution is indistinguishable from the fit in Fig. 3 and is not repeated in a separate figure). The components' relative luminosities in $V$ are computed as $79.2 \%, 10.8 \%$ and $10.0 \%$ for the primary, secondary, and tertiary, respectively, and were constrained by the Hipparcos/Tycho $V$ and $B-V$ measurement of component B, the unspotted $V$ and $B$ combined magnitude of $\mathrm{Aa}+\mathrm{Ab}+\mathrm{B}$ from APT observations, and the $V$ and $B$ combined magnitude of $\mathrm{Aa}+\mathrm{B}$ during eclipse. With the system's least spotted light of $V=7^{\text {m992 }}$ in 1993/1994 (Fig. 2), the primary's true unspotted and de-reddened magnitude becomes $V=8$. 102 . The mean residual for the $V$-band fit was insignificantly different for the two types of solution (SIM vs. REC) and was $1.5 \mathrm{mmag}$ with a standard-deviation residual of $0.5 \mathrm{mmag}$. This agrees well with our mean external precision of the (combined) $V$-band data from the APTs. The primary's radial velocities were fitted to a mean residual of $0.016 \mathrm{~km} \mathrm{~s}^{-1}$ with a standard-deviation residual of $0.13 \mathrm{~km} \mathrm{~s}^{-1}$. Those for the secondary velocities are $0.74 \mathrm{~km} \mathrm{~s}^{-1}$ and $0.34 \mathrm{~km} \mathrm{~s}^{-1}$, respectively. These results are in excellent agreement with the independent double lined orbital solution in Sect. 4.

\subsection{Spot modelling}

Two cool spots on the primary were required to fit the out-ofeclipse VI light curves. During four seasons the light-curve appeared double humped with two different minima (1997, 1998, 2000, and 2001) and was single humped during the remaining seasons.

Spot fitting was performed with SPOTMODEL (Ribarik et al. 2003), based on the rectified light curves described in the previous section. For all seasons the astrophysical properties of the triple were kept fixed at the final values in the REC column of Table 3. Since the rectification included the ellipticity effect, the spot modelling could be performed with a spherical component. This simplified the numerical treatment significantly, although we have used elliptical geometry for reconstructing stellar surface images on another occasion (Kővári et al. 2006). Two passbands were always analyzed simultaneously. Whenever T7 VI data were available, we preferred VI over T3 $B V$ because the T3 $B$ data are significantly noisier (rms of 0.01 mag compared to $0.0035 \mathrm{mag}$ in $V$ and 0.006 mag in $I$ for T7). The free parameters were the spot radii and the longitudes and latitudes. The spot temperatures were the same and were fixed at $\Delta T$ (photosphere minus spot) of $810 \mathrm{~K}$ (see previous section) if no $I$-band data were available.

Table 4 lists the results from the annual $V$ or $V I$ fits, whichever was available. Figure 5 shows the variations of the annually phased light curves and the fits with the REC binary solution and the SPOTMODEL two-spot solution. Note that these spot solutions (maps) are not mathematically unique because, firstly, light curves are just one-dimensional data, secondly, one can not distinguish spot placement between the two hemispheres due to the equator-on view and, thirdly, purely circular spots are 
Table 4. Two-spot solutions for the annual BVI light curves.

\begin{tabular}{llllllllll}
\hline \hline Year & $\ell_{\mathrm{N}}$ & $b_{\mathrm{N}}$ & $r_{\mathrm{N}}$ & $A_{\mathrm{N}}$ & $\ell_{\mathrm{S}}$ & $b_{\mathrm{S}}$ & $r_{\mathrm{S}}$ & $A_{\mathrm{S}}$ & $T_{\text {spot }}$ \\
\hline 1988.87 & 461.73 & -46.51 & 44.07 & 14.07 & 606.43 & 87.81 & 59.41 & 24.56 & $\ldots$ \\
1989.88 & 468.21 & -66.34 & 55.90 & 21.96 & 597.52 & 88.22 & 61.04 & 25.79 & $\ldots$ \\
1990.95 & 326.78 & -59.98 & 52.51 & 19.57 & 471.06 & 67.79 & 59.89 & 24.92 & $\ldots$ \\
1992.87 & 394.80 & -59.45 & 73.74 & 36.00 & 624.22 & 27.68 & 23.68 & 4.21 & $\ldots$ \\
1993.91 & 397.53 & -55.56 & 68.74 & 31.87 & 667.14 & 37.77 & 23.35 & 4.09 & $\ldots$ \\
1994.95 & 374.49 & -41.20 & 51.30 & 18.73 & 507.17 & 81.77 & 60.07 & 25.05 & $\ldots$ \\
1995.88 & 357.52 & -39.90 & 49.22 & 17.34 & 495.76 & 81.69 & 65.06 & 28.91 & $\ldots$ \\
1996.97 & 344.75 & -48.51 & 63.42 & 27.63 & 571.55 & 73.62 & 55.90 & 21.97 & $\ldots$ \\
1997.49 & 349.07 & -51.62 & 57.91 & 23.44 & 572.45 & 76.36 & 59.17 & 24.37 & $\ldots$ \\
1997.93 & 327.28 & -50.24 & 55.26 & 21.51 & 487.61 & 72.02 & 61.29 & 25.98 & 3905 \\
1998.81 & 281.78 & -67.14 & 53.28 & 20.10 & 446.18 & 78.55 & 59.82 & 24.86 & 3621 \\
1999.88 & 216.45 & -57.36 & 55.44 & 21.63 & 359.67 & 84.89 & 50.50 & 18.19 & 3562 \\
2000.92 & 181.45 & -52.35 & 40.46 & 11.95 & 383.74 & 75.26 & 55.89 & 21.96 & $\ldots$ \\
2001.90 & 144.98 & -66.31 & 48.27 & 16.71 & 316.37 & 73.70 & 51.75 & 19.04 & 3700 \\
2002.89 & 152.25 & -51.86 & 31.27 & 7.26 & 281.15 & 72.22 & 62.99 & 27.29 & 3478 \\
2003.85 & 171.40 & -66.72 & 48.04 & 16.57 & 254.50 & 76.60 & 58.11 & 23.58 & 3769 \\
2004.86 & 151.80 & -56.38 & 51.20 & 18.67 & 281.85 & 83.96 & 50.71 & 18.33 & $\ldots$ \\
2005.87 & 140.83 & -70.25 & 71.49 & 34.12 & 206.94 & 83.39 & 37.91 & 10.55 & 3899 \\
2006.91 & 259.85 & -85.79 & 67.88 & 31.17 & 266.99 & 87.82 & 40.09 & 11.75 & $\ldots$ \\
\hline
\end{tabular}

Note: index $N$ indicates the north polar cap, $S$ the south polar cap. Longitude, $\ell_{\mathrm{N} / \mathrm{S}}$, is counted from 0 (a time of maximum positive radial velocity of the primary according to Table 2 ) to $360^{\circ}$ in the same direction as the orbital motion. Latitude, $b_{\mathrm{N} / \mathrm{s}}$, is counted from $0=$ equator to $\pm 90^{\circ}=$ poles; radius, $r_{\mathrm{N} / \mathrm{S}}$, in degrees (circular spots); fractional area, $A_{\mathrm{N} / \mathrm{S}}$, in per cent of the entire stellar surface and the spot temperature ( $\left.T_{\mathrm{spot}}\right)$ in Kelvin (for light-curves with good VI data; note that the photospheric temperature is $4500 \mathrm{~K}$ ). Individual spot temperatures are assumed to be identical.

likely unrealistic. Despite these limitations, we are confident that these solutions still allow us to recover time-dependent phenomena like the fractional surface spot coverage, activity cycles (e.g. Oláh 2007; Strassmeier 2005; Lanza et al. 2006) or even differential rotation (e.g. Messina \& Guinan 2003; Strassmeier \& Oláh 2004; Walker et al. 2007). In favorable cases, our spot models may also allow the detection of active longitudes and possibly flip flops, if present (e.g. Järvinen et al. 2005; Oláh et al. 2006; Savanov \& Strassmeier 2008), but see also Jeffers (2005) for recent concerns based on simulations of artificial photometry and reconstructed active longitudes in eclipsing binaries.

\section{Discussion}

\subsection{Spot properties on the primary star}

Figure 5 summarizes our spot-model results. We emphasize that all reconstructions required just two cool polar caps, one at each rotational pole; maybe with the exception of the two seasons with the brightest ever observed magnitudes (1992/1993 and 1993/1994). Both caps appear as long lived as we have data (18 seasons spanning 19 years). Note that the SPOTMODEL solutions for 1992/1993 and 1993/1994 seem qualitatively different than in the other years, but this is simply because the brightest magnitude observed in 1993 is also the "unspotted" magnitude, i.e. in our case likely the least spotted magnitude. Thus, we implicitly forbid a spot that is permanently in view for all orbital phases during these two seasons, which may or may not be correct. In any case, it is the simplest solution and the large amplitudes of up to 0.3 in $V$, and 0.1 in $V-I$, together with an overall system dimming of up to 0.2 , can readily be accounted for with two large circum-polar spots. Their longitudes (of spot center), $\ell$, change systematically with time, as shown in the left panel in Fig. 5c, and appear to wander around the rotational poles according to the (linear) fit

$\ell_{\text {spot } \mathrm{N}}=-0.0491 \mathrm{JD}+2788.74$,

$\ell_{\text {spot } \mathrm{S}}=-0.0649 \mathrm{JD}+3733.59$.
Table 5. Final adopted astrophysical properties of HD $6286 \mathrm{Aa}, \mathrm{Ab}$, and $\mathrm{B}$.

\begin{tabular}{llll}
\hline \hline Parameter & Primary & Secondary & Tertiary \\
\hline$V_{0}, \mathrm{mag}$ & 8.102 & 10.263 & 10.35 \\
$B_{0}, \mathrm{mag}$ & 9.076 & 10.853 & 10.93 \\
$I_{0}, \mathrm{mag}$ & 6.908 & 9.587 & 9.51 \\
$(B-V)_{0}$ & 0.974 & 0.59 & 0.58 \\
Classification & $\mathrm{K} 1 \mathrm{III}$ & F6V-IV & $(\mathrm{G} 0 \mathrm{~V})$ \\
Temperature, $\mathrm{K}$ & $4500 \pm 70$ & $6300 \pm 100$ & $(6000)$ \\
Radius, $R_{\odot}$ & $12.0 \pm 0.7$ & $1.9 \pm 0.1$ & $\ldots$ \\
Luminosity, $L_{\odot}$ & $52.9_{-9}^{+10}$ & $5.1_{-0.8}^{+0.9}$ & $\ldots$ \\
Gravity, $\log g / g_{\odot}$ & $2.5 \pm 0.07$ & $4.0 \pm 0.1$ & $\ldots$ \\
Mass, $M_{\odot}$ & $1.56 \pm 0.03$ & $1.31 \pm 0.02$ & $\ldots$ \\
Metallicity, $[\mathrm{M} / \mathrm{H}]$ & solar & $\ldots$ & $\ldots$ \\
Age, Gyr & 2.7 & $\ldots$ & $\ldots$ \\
\hline
\end{tabular}

Note: The Aa-Ab pair is the eclipsing binary. The $\mathrm{B}$ component was fixed to its dereddened and transformed Hipparcos/Tycho $B-V$. Its values are poorly constrained and the values in parentheses are estimated. The index 0 indicates unspotted and dereddened values.

Here, $N$ means the north polar cap and $S$ the south polar cap (rather arbitrarily of course). JD is the fractional Julian Date (minus 2400000 ). Both central longitudes are decreasing with time, i.e. lagging behind the orbit at slightly different rates; the $N$-spot lags one rotation in $20.0 \mathrm{yrs}$, the $S$-spot, one in $15.2 \mathrm{yrs}$.

Our spot solutions remain model dependent because we fit the light and color curves under the circular cool-spot restriction. If we allow warm and cool spots to coexist, as on the Sun, the solutions become even more non-unique but would still be driven by the assumption of an immaculate magnitude. Furthermore, we excluded any features, warm or hot, on the secondary Ab component (as well as on the B tertiary). While small cool spots, peppered over the entire Aa surface could not explain by themselves the largest amplitudes observed (1992/1993 and $1993 / 1994)$, such a model would be an equally good solution for most other seasons. We emphasize that our spot solutions, although precise and consistent, are not a detailed accurate 

a) V-light curves
b) Spot model
c) Spot parameters
d) Amplitude

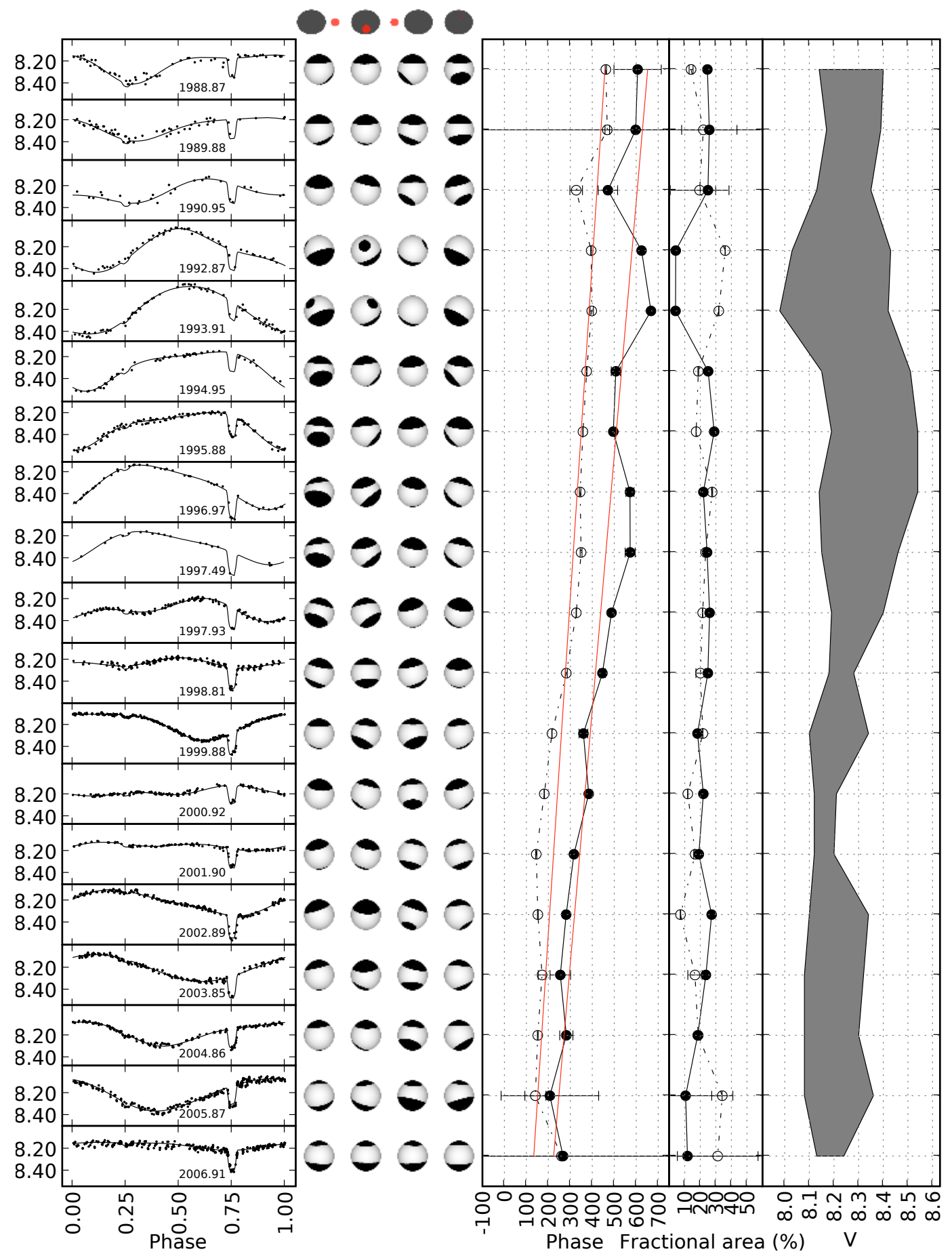

Fig. 5. Spot parameters versus time. Time increases from top to bottom. a) Annual light-curve variations during 18 observing seasons of $V$-band photometry (top to bottom) and the combined fits with the final binary model and the seasonal spot models. Note that the 1997/1998 observing season was modelled in two parts due to rapid changes in the spot distribution. b) A graphical presentation of the seasonal spot models (based on $V$ and $I$ ). Two spots are always sufficient to explain the light curves. The upper spot cap is dubbed the north $(N)$ spot, the lower cap the south $(S)$ spot. c) Spot parameters versus time (actually versus solution number because of the missing 1991/1992 season and the two solutions in the $1997 / 1998$ season). Its left panel plots the longitude each spot center ( $N$ as filled dots, $S$ in open circles) in units of rotational phase expressed as surface longitude in degrees. The drift reflects the average difference of $0.5 \%$ between the orbital period and the rotation period of the primary. Note that error bars for spot longitudes at very high latitudes are in a relative sense. The right panel plots the spot size versus time in units of the entire surface area. d) The spot-induced change of the $V$-amplitude, and the minimum and maximum brightness, are indicated in this panel. Minimum spottedness likely occurred in late 1993.

representation of the real stellar surface structure. Therefore, we refrain from further conclusions concerning the spot behavior. However, at times there is no space left on the stellar surface where our code could place large cool (or warm) spots to explain the light-curve asymmetries without additionally violating the eclipse binary geometry. Therefore, the polar regions remain 
the only places that could fit all the data, and we are confident that most of the surface activity on HD 6286 must happen consistently at or near its primary's rotational poles; and that for at least the past 19 years.

\subsection{Basic astrophysical properties}

We searched the literature and examined our own data for the brightest known visual magnitude and corresponding $B-V$ of HD 6286. From the APT data HD 6286 was brightest in 1993, with a $V$ magnitude of 7 m.992. Taking into account the magnitude differences of the visual component as well as the spectroscopic binary pair, our final adopted least-spotted $V$ magnitude of the primary is 8.102 (Table 5). O'Neal et al. (1996) showed that on some heavily spotted stars the observed maximum $V$ magnitude can underestimate the brightness of the unspotted star by $0.3-0.4$ mag. Nevertheless, we have adopted the historical maximum as the unspotted $V$ magnitude of the primary since for HD 6286 we are unable to determine a specific correction. This magnitude, combined with the Hipparcos parallax of $0 .^{\prime} 00464 \pm 0.00205$ (ESA 1997), results in $M_{V}$ (Aa) $=+1.4_{-1.3}^{+0.8}$. At a distance of $215_{-66}^{+171} \mathrm{pc}$ it is only moderately affected by interstellar extinction. Thus, we have adopted the mean extinction value of Henry et al. (2000) for the absolute magnitude calculation $\left(A_{V}=0.8 \mathrm{mag} \mathrm{kpc}^{-1}\right.$ and $\left.E(B-V)=A_{V} / 3.3\right)$.

The primary $(B-V)_{0}$ would give, in conjunction with Table 3 of Flower (1996), a bolometric correction of -0.482 and an effective temperature of $4676 \mathrm{~K}$ with an external uncertainty of certainly more than the usual $\approx 100 \mathrm{~K}$ (due to residual contamination, spots, etc.). Note that the REC solution with Nightfall suggests a lower and much better constrained value of $4500 \pm$ $70 \mathrm{~K}$, although the errors of the two determinations are such that the temperature estimates overlap. Note also that the temperature from the REC solution is a mean surface temperature rather than a photospheric temperature because of the spots. Nevertheless, the $T_{\text {eff }}$ from the $B-V$ calibration, combined with the parallax, produces a stellar luminosity of $L=25_{-13}^{+59} L_{\odot}\left(M_{\text {bol }}=1\right.$. 2$)$ and a radius of $R=7.7_{-23}^{+6.6} R_{\odot}$. The uncertainties in the computed quantities are dominated by the uncertainty in the parallax and to a lesser extent in the effective temperature. If the unspotted $V$ magnitude were 0.2 mag brighter than our adopted value, the luminosity would be increased by $20 \%$ and the radius by $10 \%$. These crude $T L R$ values are still in agreement with our independently derived values from the combined photometric and spectroscopic solution in Sect. 5, just because the error bars are so large. Table 5 summarizes the components' properties.

The minimum radius of the primary, computed from $v \sin i=$ $18.1 \mathrm{~km} \mathrm{~s}^{-1}$ and a mean rotation period equal to the orbital period of 35.67 days, is $12.8 \pm 0.7 R_{\odot}$. This suggests that the total eclipse is indeed very off center because the sum of the radii from the eclipse width, $R_{\mathrm{Aa}}+R_{\mathrm{Ab}}=9.0 R_{\odot}$, would be even smaller than the minimum radius of the primary. However, the minimum radius is still $66 \%$ larger than the radius determined from the Hipparcos parallax, but due to the large parallax error the two values are formally in agreement. Radius discrepancies between $v \sin i$-computed radii and Hipparcos-based radii have been found for several other chromospherically active giants, and Fekel et al. (1999) discussed possible sources of the problem, significant differential rotation being one. In any case, $R_{\mathrm{Aa}}+R_{\mathrm{Ab}}$ must be greater than $a \cos i$ because we detect eclipses. If $R_{\mathrm{Aa}} \approx 12.8 R_{\odot}$ and $R_{\mathrm{Ab}} \approx 2 R_{\odot}$, we would get a reasonable lower limit for the inclination of $\approx 77^{\circ}$, consistent with the partial eclipse value that we actually obtained from the combined

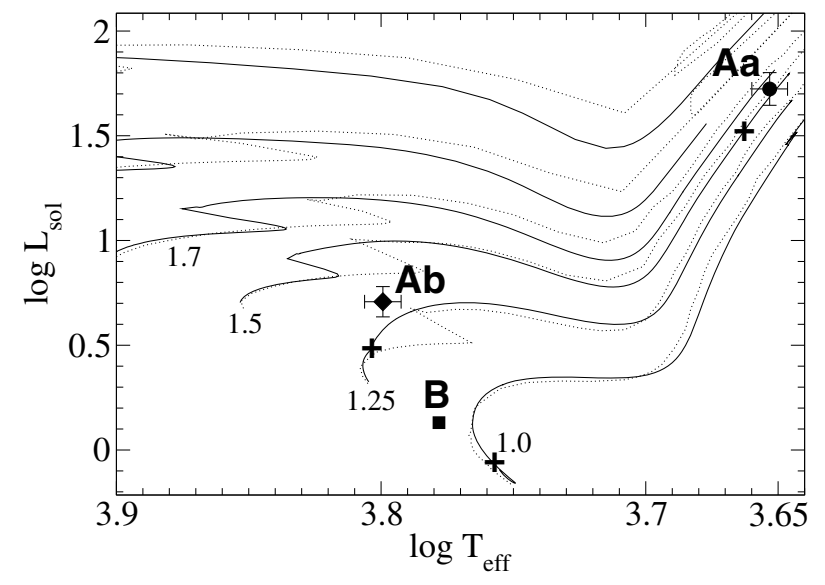

Fig. 6. The $\mathrm{K}$ giant (Aa) and F-dwarf/subgiant $(\mathrm{Ab})$ components compare to the theoretical evolutionary tracks of T. Granzer (2006, private communication; full lines) and Schaller et al. (1992; dotted lines). Note that the Schaller et al. track are shown only for comparison purpose because of their outdated opacities. The third star (B) is shown for purposes of completeness, it's position is based on the Hipparcos/Tycho color and brightness. The A components' positions are in agreement with an isochrone of age $\approx 2.7 \mathrm{Gyr}$ (as indicated by the plusses for masses of $1.0,1.25$, and $1.5 M_{\odot}$ ).

light-curve and radial-velocity solution in Sect. 5 (and listed in Table 3).

\subsection{Evolutionary status and lithium abundance}

Figure 6 compares the positions of the three components of HD 6286 with theoretical evolutionary tracks. We have computed new post main sequence evolutionary tracks with the Kippenhahn code that includes up-to-date input physics (as described in Granzer et al. 2000). No overshooting was assumed. The tracks published by Schaller et al. (1992) are shown for comparison purpose only because they were published with nowadays out-of-date opacities. All tracks are for solar metallicity. The positions of the eclipsing binary components indicate masses of approximately $1.6 M_{\odot}\left(\mathrm{K} 1\right.$ giant) and $1.3 M_{\odot}$ (F6 dwarf/subgiant), in excellent agreement with our derived values, and are best fit by an isochrone of 2.72 Gyr. The tertiary is just constrained by its Tycho $V$ and $B-V$ value but is in agreement with the isochrone fit.

The K giant appears on the red-giant branch (RGB) and close to the end of the first Li dredge-up phase. During the first dredgeup, the Li abundance at the surface gets diluted as Li-free material from the stellar interior is brought to the surface. This implies that the $\mathrm{K}$ giant primary should have a comparably low $\mathrm{Li}$ abundance, i.e. that it is not a Li rich star. The F6 star just left the main sequence or is doing so right now, while the tertiary G0 star is still on the main sequence.

Figure 7 shows three $R \approx 120000$ spectra centered at the Li I 6708-Å line. Unfortunately, all three spectra were taken during a phase close to conjunction so that any lithium line contribution from the secondary would appear blended. HD $6286 \mathrm{Aa}$ has a moderately strong lithium line with a combined equivalent width of $84 \pm 3 \mathrm{~m} \AA$, determined from a Gaussian fit from the spectrum as observed. Taking into account that the primary contributes $81 \%$ of the continuum at this wavelength increases its true line equivalent width to $104 \mathrm{~m} \AA$. This still includes a significant Fe I and VI blend and some weaker CN lines totalling $\approx 29 \mathrm{~m} \AA$ of the width of the $\mathrm{K}$ giant's blended lithium 


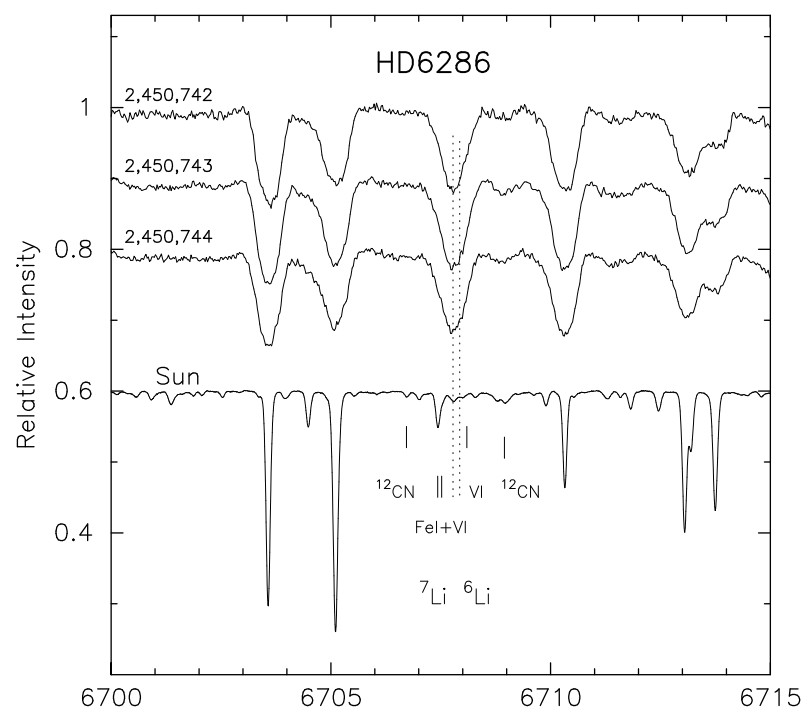

Fig. 7. Shown are three high-resolution lithium spectra of HD $6286 \mathrm{ob}-$ tained at CFHT on three consecutive nights in 1997 October (from top to bottom, phases $0.735,0.762,0.790$, appropriately shifted). All three spectra were taken near a time of conjunction and so are at single-lined phases. A very high-resolution spectrum of the Sun from the National Solar Observatory solar atlas (Kurucz et al. 1984) is shown for comparison. HD 6286 Aa shows a significant lithium line with an equivalent width of $75 \mathrm{m \AA}$.

line. After subtraction of a shifted and broadened spectrum of the G8 giant $11 \mathrm{LMi}$, obtained with the same instrumental setup, the lithium equivalent width of HD $6286 \mathrm{Aa}$ is $75 \mathrm{~m} \AA$. The non-LTE curves of growth of Pavlenko \& Magazzú (1996) for a model with $4500 \mathrm{~K}$ and $\log g=2.5$ convert this equivalent width to a logarithmic lithium abundance of 1.30 on a scale with $\log n(\mathrm{H})=12.00$. We consider this an upper limit because it is affected by the spectral type mismatch with $11 \mathrm{LMi}$ (may have a $\approx 5 \mathrm{~m} \AA$ impact on the extracted equivalent width), the spectrum subtraction uncertainties, and the assumption, probably incorrect, that the (weak) F6 secondary shows no lithium at all.

The luminosity and temperature values place the primary at or very near the RGB bump. Charbonnel \& Balachandran (2000) identified two distinct evolutionary episodes on the RGB in which extra mixing of Li may cause an unusual high surface abundance. Both episodes are related to the close vicinity of the bottom of the convective envelope to the hydrogen-burning shell. The K giant in HD 6286 has an upper limit abundance that is about a factor 8-10 below what is considered to be a lithiumrich star but appears to be a factor 10 higher than the average normal Li surface abundance (De Medeiros et al. 2000).

\subsection{Circularization and synchronization}

It is well known (e.g. Tassoul \& Tassoul 1996) that tidal interactions affect the rotational and orbital characteristics of close binaries causing them to tend toward a state in which the rotational axes of the components are parallel to the orbital axis and their rotational velocities are synchronized with the orbital period. In addition, tidal dissipation of energy causes a binary to circularize its orbit. Indeed, observational results indicate that many binaries have fully accomplished these feats. Other systems with weaker tidal interactions may not be currently old enough to have reached complete synchronization or circularization. However, these conditions may occur once tidal interactions have acted long enough or after a system evolves to a state in which tidal interactions are enhanced.

For stars with convective envelopes, Zahn $(1977,1989)$ investigated the effects of the equilibrium tide on synchronization and circularization, while Tassoul $(1987,1988)$ explored the theory that binary synchronization and circularization result because of distortions that cause large-scale hydrodynamic currents. Although these two theories disagree significantly on absolute time scales, both predict that synchronization should occur before circularization.

Chromospherically active stars have light variations caused by star spots rotating in and out of view, enabling the rotation period of the spotted star to be determined. The F6 secondary with its thin convective envelope is not expected to generate significant chromospheric and photospheric activity. Thus, the rapidly rotating K-giant primary is clearly the dominant source of the star spot light variability. The photometric periods of 35.3 days (Hooten \& Hall 1990) and 35.53 days (ESA 1997), and 35.49 days (this paper, Sect. 5.2) are, therefore, measurements of the rotation period of the $\mathrm{K}$ giant at the latitudes where there were spots during the times of these observations. Since the binary period is 35.671 days, the $\mathrm{K}$ giant is rotating synchronously to within about $0.5 \%$. Such synchronization of the chromospherically active component is typical of the vast majority of systems with orbital periods up to 30 days (Fekel \& Eitter 1989), and HD 6286 has a period just slightly greater than this value. Furthermore, surface-latitude dependent differential rotation will smear out the true rotational period from photometric time series. Deviations from synchronous rotation of up to, say, $10 \%$ could be accounted for by such differential rotation (Walker et al. 2007; Strassmeier \& Bopp 1992, a.o.).

From its radius of $1.9 R_{\odot}$ and an orbital period of 35.67 days, we computed the synchronous velocity of the F6-dwarf/subgiant to be $2.7 \mathrm{~km} \mathrm{~s}^{-1}$. In several spectra of HD 6286 we measured the full width at half-maximum of the one or two least blended metal lines of the F6 component. Following the procedure of Fekel (1997), the mean line broadening for each spectrum was converted into a $v \sin i$ value. If the orbital and rotational axes are parallel, a condition expected and generally assumed for close binaries, then the inclination is the same. Because HD 6286 is an eclipsing system, our $v \sin i$ value is essentially the equatorial rotational velocity and can be directly compared to the computed synchronous velocity. From five spectra our measured rotational velocity of the F6 dwarf/subgiant ranges from 7 to $13 \mathrm{~km} \mathrm{~s}^{-1}$. This range of values is likely to be an upper limit, however, because even the best lines of the F6 star are very weak and usually partially blended with weak lines of the K giant. Nevertheless, the computed synchronous velocity is 3 to 5 times smaller than our measured values (an academic guestimate), and so synchronous rotation is possible, but can not be conclusively established. We note that the F6 dwarf/subgiant is very slowly rotating compared to other single stars of that spectral type.

Our photometric results indicate that HD 6286 has a circular orbit. The circularization time scale, like the synchronization time scale, is principally dependent on the ratio of the semimajor axis of the relative orbit, $a$, to the stellar radius, $R$. Thus, the rapid increase in the size of the $\mathrm{K}$ giant, as it evolved across the Hertzsprung gap and up the giant branch, likely caused the orbit to become circular.

Acknowledgements. Lou Boyd's dedicated efforts at Fairborn Observatory have been invaluable. We are grateful to Dr. Doug Hall for helping to intiate the work on this star. We thank Drs. Prsa and Wichmann for providing us with copies of the PHOEBE and the Nightfall programs, respectively. The automated astronomy program at Tennessee State University has been supported in part by grants 
from NASA and NSF and at AIP by the German State of Brandenburg. We thank our colleague Dr. Thomas Granzer for computing the evolutionary tracks for us.

\section{References}

Abt, H. A., \& Morrell, N. I. 1995, ApJS 99, 135

Barden, S. C. 1985, ApJ 295, 162

Barker, E. S., Evans, D. S., \& Laing, J. D. 1967, R. Obs. Bull., 130

Batten, A. H., Fletcher, J. M., \& MacCarthy, D. G. 1989, Publ. Dom. Astrophy. Obs., 17, 1

Bidelman, W. P. 1983, AJ, 88, 1182

Boesgaard, A. M., \& Tripicco, M. J. 1986, ApJ, 303, 724

Charbonnel, C., \& Balachandran, S. C. 2000, A\&A, 359, 563

Couteau, P. 1970, A\&AS, 1, 419

Crews, L. J., Hall, D. S., Fekel, F. C., \& Henry, G. W. 1996, International Amateur-Professional Photoelectric Photometry Communication, 65, 43

Dempsey, R. C., Linsky, J. L., Fleming, T. A., \& Schmitt, J. H. M. M. 1997, ApJ, 478,358

De Medeiros, J. R., do Nascimento, J. D., Sankarankutty, S., Costa, J. M., \& Maia, M. R. G. 2000, A\&A, 363, 239

ESA 1997, The Hipparcos and Tycho Catalogues, ESA SP-1200

Fabricius, C., \& Makarov, V. V. 2000, A\&A, 356, 141

Fekel, F. C. 1997, PASP, 109, 514

Fekel, F. C., \& Eitter, J. J. 1989, AJ, 97, 1139

Fekel, F. C., Strassmeier, K. G., Weber, M., \& Washuettl, A. 1999, A\&AS, 137, 369

Fitzpatrick, M. J. 1993, in Astronomical Data Analysis Software and Systems II, ed. R. J. Hanisch, R. V. J. Brissenden, \& J. Barnes (San Francisco: ASP), ASP Conf. Ser., 52, 472

Flower, P. J. 1996, ApJ, 469, 355

Granzer, T., Reegen, P., \& Strassmeier, K. G. 2001, AN, 322, 325

Granzer, T., Schüssler, M., Caligari, P., \& Strassmeier, K. G. 2000, A\&A, 355, 1087

Heard, J. F. 1956, Publ. David Dunlap Obs., 2, 107

Henry, G. W., Fekel, F. C., Henry, S. M., \& Hall, D. S. 2000, ApJS, 130, 201

Henry, G. W., Fekel, F. C., \& Henry, S. M. 2005, AJ, 129, 2815

Hooten, J. T., \& Hall, D. S. 1990, ApJS, 74, 225

Huenemoerder, D. P., \& Barden, S. C. 1984, AAS, 16, 510

Järvinen, S., Berdyugina, S., \& Strassmeier, K. G. 2005, A\&A, 440, 735

Jeffers, S. 2005, MNRAS, 359, 729

Johnson, H. L., \& Morgan, W. W. 1953, ApJ, 117, 313

Kazarovets, E. V., Samus, N. N., \& Goranskij, V. P. 1993, Inf. Bull. Var. Stars, 3840
Keenan, P. C., \& McNeil, R. C. 1989, ApJS, 71, 245

Kolláth, Z. 1990, The program package MUFRAN, Occasional Technical Notes of Konkoly Observatory, 1 ,

wWw. konkoly.hu/Mitteilungen/...\#TechNotes

Kővári, Zs., Bartus, J., Strassmeier, K. G., et al. 2006, A\&A, 463, 1071

Kurucz, R. L., Furenlid, I., Brault, J., \& Testerman, L. 1984, NSO Atlas No. 1, Solar flux atlas from 296 to $1300 \mathrm{~nm}$

Lanza, A. F., Piluso, N., Rodonó, M., Messina, S., \& Cutispoto, G. 2006, A\&A, 455,595

Lucy, L. B., \& Sweeney, M. A. 1971, AJ, 76, 544

Messina, S., \& Guinan, E. F. 2003, A\&A, 409, 1017

Oláh, K. 2007, IAU JD9, Prague General Assemby

Oláh, K., Budding, E., Kim, H.-I., \& Etzel, P. B. 1994, A\&A, 291, 110

Oláh, K., Korhonen, H., Kővári, Zs., Forgacs-Dajka, E., \& Strassmeier, K. G. 2006, A\&A, 452, 303

O’Neal, D., Saar, S., \& Neff, J. E. 1996, ApJ, 463, 766

Pasquini, L., \& Lindgren, H. 1994, A\&A, 283, 179

Pavlenko, Ya. V., \& Magazzú, A. 1996, A\&A, 311, 961

Pearce, J. A. 1957, Trans. IAU, 9, 441

Prsa, A., \& Zwitter, T. 2005, ApJ, 628, 426

Ribárik, G., Oláh, K., \& Strassmeier, K. G. 2003, AN, 324, 202

Savanov, I., \& Strassmeier, K. G. 2008, AN, 329, 364

Scarfe, C. D., Batten, A. H., \& Fletcher, J. M. 1990, Publ. Dominion Astrophys. Obs. Victoria, 18, 21

Schaller, G., Schaerer, D., Meynet, G., \& Maeder, A. 1992, A\&AS, 96, 269

Strassmeier, K. G. 2005, AN, 326, 269

Strassmeier, K. G., \& Fekel, F. C. 1990, A\&A, 230, 389

Strassmeier, K. G., \& Bopp, B. W. 1992, A\&A, 259, 183

Strassmeier, K. G., \& Rice, J. B. 1998, A\&A, 339, 497

Strassmeier, K. G., \& Oláh, K. 2004, ESA SP-583, 149

Strassmeier, K. G., Hall, D. S., Fekel, F. C., \& Scheck, M. 1993, A\&AS, 100, 173

Tassoul, J.-L. 1987, ApJ, 322, 856

Tassoul, J.-L. 1988, ApJ, 324, L71

Tassoul, J.-L., \& Tassoul, M. 1996, Fund. Cosmic Phys., 16, 377

Taylor, B. J. 1999, A\&AS, 134, 523

Taylor, B. J. 2003, A\&A, 398, 731

Walker, G. A. H., Croll, B., Kuschnig, R., et al. 2007, ApJ, 659, 1611

Wichmann, R. 2006, http: //la-samhna.de/nightfall/

Wolfe, R. H., Horak, H. G., \& Storer, N. W. 1967, in Modern Astrophysics, ed. M. Hack (New York: Gordon \& Breach), 251

Zahn, J.-P. 1977, A\&A, 57, 383

Zahn, J.-P. 1989, A\&A, 220, 112 